Enferm Bras 2020;19(4);329-35

https://doi.org/10.33233/eb.v19i4.4218

\title{
ARTIGO ORIGINAL \\ Indicador de qualidade em hospital de ensino: características do cancelamento de cirurgias eletivas
}

Jaqueline Lopes Gouveia*, Marli de Carvalho Jericó, D.Sc. ${ }^{\star *}$, Priscila Buck de Oliveira Ruiz ${ }^{* \star *}$, Paula Buck de Oliveira Ruiz ${ }^{\star \star \star *}$, Renata Prado Bereta Vilela, M.Sc. ${ }^{* \star \star \star}$, Dalva Maria da Silveira Roland, D.Sc. ${ }^{* \star * \star *}$, Ângela Silveira Gagliardo Calil, M.Sc. ${ }^{* \star \star \star \star *}$

${ }^{\star}$ Enfermeira, especialização em Centro Cirúrgico, Recuperação Anestésica e Central de Materiais e Esterilização pela Faculdade de Medicina de São José do Rio Preto, Enfermeira clínica do Centro Cirúrgico do Hospital de Base de São José do Rio Preto, ${ }^{\star \star} E n f e r m e i r a$, Professora do curso de graduação em Enfermagem da Faculdade de Medicina de São José do Rio Preto, ${ }^{* * \star}$ Enfermeira, Mestranda do Curso de pós graduação Stricto Sensu, pela Faculdade de Medicina de José do Rio Preto/SP, Enfermeira coordenadora do bloco operatório do Hospital de Base de São José do Rio Preto, ${ }^{* * * *}$ Enfermeira, doutoranda do Programa de Pós-Graduação em Gerenciamento em Enfermagem, USP/SPS, ${ }^{\star \star * * \star E n f e r m e i r a, ~ D o c e n t e ~ d o ~ c u r s o ~ d e ~ M e d i c i n a ~}$ do FACERES, São José do Rio Preto/SP, ${ }^{* * * * * E}$ Enfermeira, Doutora pela Faculdade de Medicina de São José do Rio Preto, FAMERP, Professora do curso de Graduação em Enfermagem da FAMERP, ${ }^{\star \star * \star * \star * E n f e r m e i r a, ~ M e s t r e ~ p e l a ~ F a c u l d a d e ~ d e ~ M e d i c i n a ~ d e ~ S a ̃ o ~ J o s e ́ ~ d o ~ R i o ~ P r e t o ~-~}$ FAMERP, Professora do curso de Graduação em Enfermagem da FAMERP

Recebido em 22 de junho de 2020; aceito em 20 de agosto de 2020.

Correspondência: Jaqueline Lopes Gouveia, Rua República do Líbano, 3000, apto 32, bloco 10 Tarraf II, 15092-440 São José do Rio Preto SP

Jaqueline Lopes Gouveia: jaquelinelgouveia@hotmail.com

Marli de Carvalho Jericó: marlicj2010@gmail.com

Priscila Buck de Oliveira Ruiz: pbuck@bol.com.br

Paula Buck de Oliveira Ruiz: paulabuckruiz@usp.br

Renata Prado Bereta Vilela: renata_bereta@hotmail.com

Dalva Maria da Silveira Roland: dalva@famerp.br

Ângela Silveira Gagliardo Calil: angela@famerp.br

\section{Resumo}

Introdução: $\mathrm{O}$ ato cirúrgico é um procedimento relevante para a recuperação e manutenção da saúde, uma vez que, após tal acontecimento, é esperado que o cliente evolua com uma melhor qualidade de vida. Objetivo: Investigar a taxa, causas e especialidades responsáveis pelos cancelamentos cirúrgicos. Métodos: Estudo quantitativo, retrospectivo de janeiro a dezembro de 2016, realizado em hospital de ensino. Foi adotada a fórmula proposta pelo Compromisso com a Qualidade Hospitalar (CQH). Resultados: O indicador de qualidade variou de 5,9\% a 11,5\%. As principais causas de cancelamentos relacionados à instituição foram divididas entre o Sistema Único de Saúde (SUS) que apresentou a falta de tempo hábil $(38,7 \%$ ) e a troca pela equipe entre pacientes devido à gravidade $(16,1 \%)$ e o Sistema de Saúde Suplementar (SSS), com a não autorização pela operadora de saúde $(55,1 \%)$. Em relação aos motivos dos pacientes encontrouse no SSS e SUS a falta de internação (SUS - 26,4\% e SSS - 29,3\%) e falta de condições clínicas do paciente (SUS - $25 \%$ e SSS - 18,4\%). Em relação a especialidade médica, $83,3 \%$ não foi identificada, seguida da equipe da ortopedia com 4,1\%. Conclusão: O indicador de cancelamento cirúrgico mostrou-se elevado nesse período do estudo, quando comparado a $\mathrm{CQH}$, e a especialidade foi ortopedia.

Palavras-chave: centro cirúrgico, suspensão de tratamento, enfermagem de centro cirúrgico.

\section{Abstract}

Quality indicator in a teaching hospital: characteristics of elective surgery cancellation Introduction: It is essential to measure surgical cancellation for health services, as it influences the client's health and the operational unit. Objective: To investigate taxa, motives and specialties involved in surgical cancellations. Methods: A quantitative, retrospective study from January to 
December 2016, carried out at a teaching hospital. It was adopted the formula proposed by the Commitment to Hospital Quality (CQH). Results: The quality indicator ranged from 5.9\% to $11.5 \%$. The main reasons for cancellation related to institutions were lack of time (38.7\%) and priority for critically ill patients (16.1\%) for users of the Unified Health System (UHS), and for beneficiaries of the Supplementary Health System (SHS) - unauthorized agreement (55.1\%). The reasons for patients in the SHS and UHS were lack of hospitalization (UHS - 26.4\% and SHS - 29.3\%) and poor patient's clinical condition (UHS - 25\% and SHS - 18.4\%). Conclusion: The surgical cancellation indicator was elevated in this study period, when compared to the $\mathrm{CQH}$, and the main specialty was orthopedics.

Keywords: surgicenters, withholding treatment, operating room nursing.

\section{Resumen}

Indicador de calidad en un hospital universitario: características de la cancelación de cirugía electiva

Introducción: Es esencial medir la cancelación quirúrgica de los servicios de salud, ya que influye en la salud del cliente y en la dinámica de la unidad. Objetivo: Investigar la tasa, los motivos y las especialidades responsables de las cancelaciones quirúrgicas. Métodos: Estudio cuantitativo retrospectivo de enero a diciembre de 2016, realizado en un hospital docente. Los datos fueron puestos a disposición por el quirófano y para el cálculo del indicador, se adoptó la fórmula propuesta por el Compromiso con la Calidad Hospitalaria (CQH). Los resultados se analizaron cuantitativamente, utilizando estadísticas descriptivas básicas. Resultados: El indicador de calidad osciló entre $5.9 \%$ y $11.5 \%$. Las principales razones de las cancelaciones relacionadas con la institución fueron: para los usuarios del Sistema Único de Salud (SUS), la falta de tiempo $(38,7 \%)$ y prioridad para los pacientes críticos $(16,1 \%)$ y los beneficiarios del Sistema de Salud Complementario (SSC) - acuerdo no autorizado (55,1\%). En cuanto a las razones de los pacientes, se encontró en el SSC y el SUS la falta de hospitalización (SUS - 26,4\% y SSC $29,3 \%$ y de condiciones clínicas del paciente. Conclusión: El indicador de cancelación quirúrgica fue alto en este período del estudio, cuando comparado con la $(\mathrm{CQH})$, y la especialidad fue la ortopedia.

Palabras-clave: centro quirúrgico, privación del tratamiento, enfermería de quirófano.

Introdução

O ato cirúrgico é um procedimento importante para a recuperação e manutenção da saúde de um indivíduo, uma vez que, após tal acontecimento, é esperado que o cliente evolua com uma melhor qualidade de vida [1]. As cirurgias podem ser de caráter diagnóstico, eletivas, emergências e de urgências, além disso, o centro cirúrgico é um ambiente que requer profissionais capacitados devido à elevada tecnologia utilizada e à variabilidade da dinâmica de trabalho [2].

A dinâmica de trabalho em uma unidade cirúrgica deve ocorrer de forma equilibrada entre todos os membros da equipe, os quais devem ser profissionais capacitados, proporcionando um melhor enfrentamento e bem-estar da própria equipe e paciente [3].

O fato de o paciente se submeter a qualquer procedimento cirúrgico, é exigido de toda a equipe um planejamento eficaz para o atendimento, sendo assim, normalmente, é esperado de todo enfermeiro, alta performance na qualidade assistencial e utilização de recursos (humanos, materiais e equipamentos) $[4,5]$.

Para atender a necessidade de mensuração da qualidade no serviço, um dos instrumentos frequentemente utilizados para essa avaliação tem sido os indicadores, que são medidas ou mensurações que ajudam a entender o tamanho, valor ou representatividade de uma variável. O monitoramento de determinados processos por meio de indicadores é uma ferramenta importante de gestão, podendo corrigir problemas e redirecionar decisões gerenciais [6].

Um dos indicadores utilizados no bloco cirúrgico com função em medir tanto a qualidade quanto a produtividade é o cancelamento cirúrgico. Este indicador é relevante para 0 gerenciamento dos serviços de saúde, uma vez que influencia diretamente no cliente e na dinâmica da unidade [7]. Sendo assim, este é definido a partir do cálculo em que número de cirurgias suspensas é dividido pelo número total de cirurgias agendadas em um determinado período na instituição e multiplicado por 100 [6].

As causas do cancelamento cirúrgico, nacionalmente, são relatadas pelos autores por causas relacionadas à estrutura hospitalar (falta de equipe anestésica) [8] e ao paciente (falta de 
pontualidade, ausência de preparo pré-operatório, instabilidade hemodinâmica, absenteísmo e outros) [1,9]. Internacionalmente, essa temática também tem sido motivo de preocupação, como mostra estudo australiano que relacionou os cancelamentos ao paciente (absenteísmo), instalações (falta de equipamento), recursos humanos (anestesista, cirurgiões) e outras razões não documentadas [4].

As consequências do cancelamento envolvem mudanças no cotidiano do cliente e familiares, pois, a partir do momento que são informados a respeito do procedimento, eles são obrigados a replanejarem suas rotinas. Além deste fato, reflete na atividade laboral dos mesmos, pois estarão, em muitos casos, incapacitados de voltarem às suas atividades produtivas [9]. Ainda, a carga emocional do cliente se mantém elevada, caracterizada por alguns comportamentos como: a ansiedade, medo e insegurança [10].

Institucionalmente, implica em vários processos, mas diretamente no planejamento e organização do procedimento, despendendo tempo, recursos materiais e humanos que geram prejuízos [10] e a não inclusão de outro cliente na programação cirúrgica acentua os gastos ao serviço [11]. Portanto, há um comprometimento de toda a agenda cirúrgica, ocasionando uma sequência de atrasos, os quais contribuem para aumentar o tempo de permanência, risco de infecção, desnutrição e custo [12].

Estudos abordando a problemática sobre cancelamento cirúrgico apontaram taxas como $6,79 \%$ [13], 27,4 [10], 30,6 [1] e 44,2\% [14]. Tal situação é considerada uma ocorrência importante, este fato contribui para o planejamento da unidade, direcionando estratégias capazes de reduzirem custos, além de diminuírem as consequências não favoráveis, causadas aos clientes e seus familiares como, por exemplo, atraso no tratamento de câncer, atraso para retirada de um cateter calcificado, entre outros motivos que justificam a importância na realização do estudo atual $[1,10,14-16]$. Neste contexto, para reduzir a taxa de cancelamento cirúrgico nas organizações de saúde, os gestores estão realizando diversas intervenções, como a busca ativa, confirmação telefônica prévia do paciente no mapa cirúrgico e consulta pré-anestésica $[10,17]$.

Dessa forma, o indicador de cancelamento cirúrgico é adotado nos hospitais como um indicador de qualidade e produtividade do serviço prestado, na busca de proporcionar a melhor assistência e segurança ao cliente. Diante de tal abordagem, pretende-se com este estudo responder a seguinte questão de pesquisa: "Qual a ocorrência, a causa e as especialidades médicas relacionadas aos cancelamentos cirúrgicos de um hospital de ensino?". Para tal, o objetivo desta pesquisa é investigar a taxa, causas e especialidades médicas relacionadas aos cancelamentos cirúrgicos de um hospital de ensino.

\section{Material e métodos}

Estudo quantitativo, de campo, transversal realizado em hospital de ensino, de porte especial (708 leitos), do sudeste brasileiro. Essa instituição presta atendimento para aproximadamente 2 milhões de habitantes de 102 municípios da Divisão Regional de Saúde de Rio Preto (DRS 15). No sistema público - aos usuários do Sistema Único de Saúde (SUS) e ao sistema privado - aos beneficiários do Sistema de Saúde Suplementar (SSS), composto por várias operadoras e seguradoras de saúde credenciadas ao hospital e atende também a pacientes particulares.

O estudo foi realizado no centro cirúrgico da instituição, que é composto por 25 salas operatórias, realizando em média 27.570 cirurgias/ano, dessas $16.156(58,23 \%)$ pelo SUS e $11.414(41,40 \%)$ pelo SSS.

Os critérios de inclusão foram compostos por cirurgias eletivas canceladas no período de janeiro a dezembro do ano de 2016 de todas as especialidades médicas e os de exclusão foram as cirurgias de urgência e emergência, obstétricas, oftalmológicas e cirurgias de outro hospital anexo especializado na saúde da criança e da mulher.

Os dados foram coletados no mês de julho de 2017, após aprovação do Comitê de Ética em Pesquisa (CEP) - parecer no 2.176.379, por meio do banco de dados do centro cirúrgico do hospital em estudo. Para cálculo do indicador de taxa de cirurgia cancelada foi aplicada a fórmula adaptada da proposta pelo $\mathrm{CQH}[6]$ :

Taxa de cancelamento cirúrgico $=\underline{\text { Número de cirurgias canceladas } \times 100}$ Total de cirurgias agendadas 
Os dados foram armazenados numa planilha eletrônica, utilizando o programa Excel e a estatística descritiva foi usada para análise dos dados. As causas dos cancelamentos foram agrupadas por categorias, sendo elas, relacionadas ao paciente e extra paciente.

Resultados

No período estudado houve 24.232 procedimentos cirúrgicos agendados, variando de 1.719 (abril) a 2.371 (agosto). O indicador de cancelamento cirúrgico apresentou taxa de $7,51 \% /$ ano, com mediana de $5,90 \%$ variando de $5,90 \%$ (novembro) a $11,51 \%$ (janeiro), como mostra a tabela I.

Tabela I - Distribuição da taxa de cancelamento cirúrgico mensal em 2016, segundo o tipo de financiamento de saúde. São José do Rio Preto, 2017.

\begin{tabular}{|c|c|c|c|c|c|c|}
\hline Mês & \multicolumn{3}{|c|}{ Cirurgias agendadas } & \multicolumn{3}{|c|}{ Cirurgias canceladas } \\
\hline & $\mathrm{N}(\%$ & & & & $N(\%)$ & \\
\hline & Total & Público & Privado & Total & Público & Privado \\
\hline Janeiro & 1.919 & 975 & 944 & $221(11,51)$ & $123(12,61)$ & $98(10,38)$ \\
\hline Fevereiro & 1.874 & 1048 & 826 & $183(9,76)$ & $102(9,73)$ & $81(9,80)$ \\
\hline Março & 1.994 & 1190 & 804 & $155(7,77)$ & $128(10,75)$ & $27(3,35)$ \\
\hline Abril & 1.719 & 1019 & 700 & $140(8,14)$ & $121(11,87)$ & $19(2,71)$ \\
\hline Maio & 1.864 & 1114 & 750 & $143(7,67)$ & $118(10,59)$ & $25(3,33)$ \\
\hline Junho & 2.126 & 867 & 1259 & $129(6,06)$ & $112(12,91)$ & $17(1,35)$ \\
\hline Julho & 2.154 & 1008 & 1146 & $124(5,75)$ & $109(10,81)$ & $15(1,30)$ \\
\hline Agosto & 2.371 & 1011 & 1360 & $148(6,24)$ & $117(11,57)$ & $31(2,27)$ \\
\hline Setembro & 2.274 & 993 & 1281 & $155(6,81)$ & $141(14,19)$ & $14(1,09)$ \\
\hline Outubro & 2.173 & 939 & 1234 & $169(7,77)$ & $146(15,54)$ & $23(1,86)$ \\
\hline Novembro & 1.879 & 737 & 1142 & $111(5,90)$ & $95(12,89)$ & $16(1,40)$ \\
\hline Dezembro & 1.885 & 919 & 966 & $142(7,53)$ & $120(13,05)$ & $22(2,27)$ \\
\hline Total & 24.232 & 11.820 & 12.412 & $1.820(7,51)$ & $1.432(12,11)$ & $388(3,12)$ \\
\hline
\end{tabular}

Foram observados cancelamentos em 636 pacientes do SUS e 267 do SSS. Em relação ao perfil traçado por causa de cancelamento cirúrgico, o agrupamento relacionado ao paciente proveniente do SUS, variou de exame alterado $22(3,45 \%)$ a falta de internação $168(26,41 \%)$ e o paciente do sistema privado variou de procedimento já realizado $17(6,36 \%)$ a não internou 78 $(29,21 \%)$ (Tabela II).

Tabela II - Distribuição das causas de cancelamento cirúrgico em relação ao paciente, segundo tipo de financiamento de saúde. São José do Rio Preto, 2017.

\begin{tabular}{lll}
\hline Cau sas & Público N $(\%)$ & Privado N (\%) \\
\hline Que bra de jejum & $78(12,27)$ & $6(2,30)$ \\
Não internou & $168(26,42)$ & $78(29,30)$ \\
Recusou a fazer a dirurgia & $37(05,81)$ & $42(15,80)$ \\
Sem condições clínicas & $159(25,00)$ & $49(18,40)$ \\
Exame alterado & $22(03,45)$ & - \\
Procedimento realizado & - & $17(6,40)$ \\
Outros & $172(27,05)$ & $74(27,80)$ \\
\hline Total & $636(100)$ & $267(100)$ \\
\hline
\end{tabular}

Quanto ao indicador de cancelamento, encontrou-se um total de 903 causas, com mediana de $3,72 \%$, relacionadas ao paciente.

$\mathrm{Na}$ tabela III, tratando-se das causas de cancelamento cirúrgico pela instituição, referente ao SUS, os dados variaram de médicos com problemas particulares $9(1,11 \%)$ a falta de tempo hábil $314(38,76 \%)$. Já em relação ao paciente do sistema privado, variou de falta de material de Centro de Material e Esterilização (CME) com $1(0,93 \%)$ a falta de autorização do convênio com 59 (55,14\%).

Ao realizar o cálculo do indicador de cancelamento referentes às causas extra paciente, foram encontrados 917 cancelamentos, com mediana de 3,78\%.

Em relação ao perfil por especialidades médicas, os cancelamentos cirúrgicos variaram de $(0,04 \%)$, relacionados às equipes da ginecologia e obstetrícia e mastologia, a $(4,10 \%)$ de ortopedia. Contudo, não foi possível, por falta de registros, a identificação da especialidade na maioria dos cancelamentos - $83,35 \%$. (Figura 1 ) 
Tabela III - Distribuição das causas de cancelamento cirúrgico extra paciente, segundo tipo de financiamento de saúde. São José do Rio Preto, 2017.

\begin{tabular}{lll}
\hline Causas & Público N (\%) & Privado N (\%) \\
\hline Alteração na escala de agendamento & $24(2,96)$ & $5(4,67)$ \\
Falta de anestesia & $23(2,83)$ & $2(1,86)$ \\
Falta de chefe da especialidade & $27(3,33)$ & - \\
Falta de disponibilidade da equipe médica & $87(10,74)$ & $5(4,67)$ \\
Falta de material da CME & $17(2,09)$ & $1(0,93)$ \\
Falta de leito de UTI & $41(5,06)$ & $2(1,86)$ \\
Falta de tempo hábil & $314(38,76)$ & $2(1,86)$ \\
Optado por outro procedimento cirúrgico & $36(4,44)$ & $4(3,73)$ \\
Optado por paciente mais grave & $131(16,17)$ & - \\
Optado por tratamento conservador & $38(4,69)$ & $9(8,41)$ \\
Falta de autorização da OPS & - & $59(55,14)$ \\
Pessoal da equipe médica & $9(1,11)$ & $10(9,34)$ \\
Outros & $63(7,77)$ & $8(7,47)$ \\
\hline Total & $\mathbf{8 1 0 ( 1 0 0 )}$ & $\mathbf{1 0 7}(\mathbf{1 0 0})$ \\
\hline
\end{tabular}

UTI = Unidade de Terapia Intensiva; OPS = Operadora de Plano de Saúde

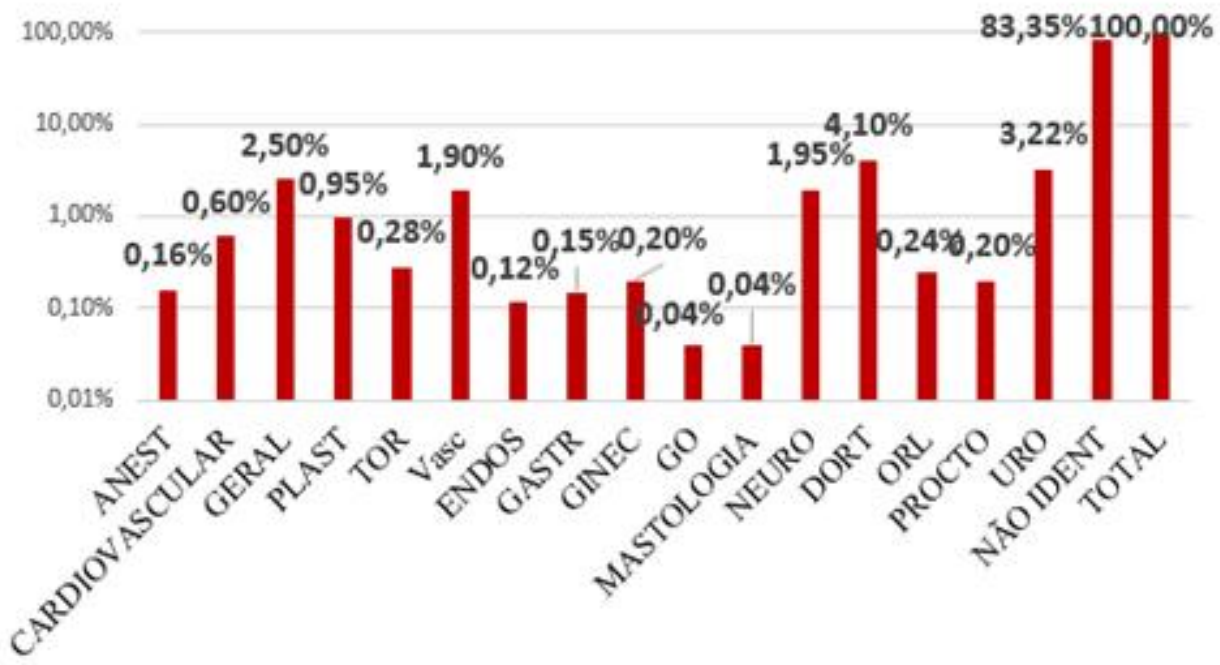

Figura 1 - Distribuição das cirurgias canceladas em relação as especialidades médicas em 2016. São José do Rio Preto, 2017.

\section{Discussão}

A presente pesquisa apresentou taxa média/ano de cancelamento cirúrgico de 7,51\%, informação essa inferior a outros estudos realizados em hospitais de ensino. Em Belo Horizonte, estudo em hospital de grande porte, que presta atendimento ao sistema de saúde público e privado, evidenciou taxa média de 5,20\% [11]. Em Fortaleza, estudo encontrou resultado superior $16,00 \%$ em um hospital público, de porte médio [17]. No sul de Minas Gerais, hospital geral de ensino, com atendimento ao SUS, apresentou taxa de 27,4\% [10]. Ainda, hospital escola de Pernambuco revelou taxa de 30,6\% [1]. Acredita-se que tal variabilidade ocorre porque existem diferentes estruturas hospitalares e diferentes formas de análise e controle dos indicadores de qualidade, demonstrando a necessidade de padronização nos dados, para uma melhor evidência e posterior tomada de decisão pelos gestores hospitalares.

Os meses com maiores taxas foram janeiro $(11,51 \%)$, seguido de fevereiro $(9,76) \mathrm{e}$ março $(7,77 \%)$. Percebe-se que são meses sazonais por várias causas, entre elas, entrada de novos residentes e férias escolares (o que poderia justificar a não internação dos pacientes). Em relação à menor taxa 5,90\% ocorreu em novembro, diferindo de estudo realizado em um hospital 
que atende, também, ao sistema público e privado que encontrou nesse período maiores taxas de cancelamento $(6,60 \%)$ e menor em agosto $(3,60 \%)$ [11].

As causas de cancelamento cirúrgico neste estudo foram divididas em "causas do paciente" e "causas extra paciente". Em um hospital de média complexidade [17], concluiu-se que as causas do paciente estavam relacionadas principalmente a "falta de condições clínicas" - 50,3\% e "não comparecimento" - 39,9\%, ou seja, pode-se dizer que possui semelhanças com a pesquisa atual, quando comparado com SUS e SSS. Já a respeito das "causas extra paciente" ou "causas da instituição", o mesmo autor evidenciou em relação ao SUS "prioridade para urgência" - 72,1\% e "erro na programação cirúrgica" - 12,5\%, os quais também possuem semelhanças com as causas descritas nessa pesquisa. Os termos que se assemelham aos mencionados acima foram a "falta de tempo hábil", também descrito como não conformidade na programação cirúrgica, o que compromete a falta de tempo para a realização de procedimento e a causa "optado por paciente mais grave" que pode ser considerado semelhante ao termo "propriedade para urgência" [17-19].

Quando comparados os totais de cancelamentos de cirurgias do SUS e SSS, podemos observar que o SUS possui maior taxa de cancelamentos com $12,11 \%$, enquanto o SSS apresenta uma taxa de 3,12\%, ou seja, aproximadamente 3,9 vezes maior que o sistema privado de saúde. Existem algumas comparações, que podem ser usadas como explicação para a grande diferença entre as taxas, sendo elas, o maior número de cirurgias pelo SUS e por ser um hospital de ensino, possui residentes, que atuam diretamente nos procedimentos cirúrgicos, causando aumento no tempo cirúrgico, os quais não atuam diretamente em cirurgias do SSS.

A maior taxa de cancelamento encontrada, neste estudo, relacionada a especialidades médicas, foi de $83,35 \%$, constituindo-se em grande viés na pesquisa. O termo "não identificado" foi encontrado por falta de preenchimento do campo "especialidade" pelos profissionais, com isso era enviado ao banco de dados e registrado como "não identificado". As especialidades médicas com maior taxa de cancelamento cirúrgico foram atribuídas às equipes de ortopedia $(4,10 \%)$ e urologia $(3,22 \%)$, dados que se assemelham a outras investigações como em um hospital de ensino, no Paraná que, também, encontrou (42,30\%) relacionadas a equipe médica da ortopedia [20] e, ainda, em estudo internacional na cidade de Buenos Aires, com $(54,00 \%)$ da equipe da Urologia [16].

Uma das limitações deste estudo está relacionada a formatação do sistema de informação hospitalar vigente que comprometeu investigar com detalhes os dados referentes às especialidades médicas constituindo-se em viés nos resultados. Contudo, gerou oportunidade de intervenção da pesquisadora em conjunto com a gestora da unidade, aplicando uma ferramenta de qualidade para solucionar essa não conformidade e gerar melhoria quanto a acurácia de identificação das especialidades médicas no sistema de informação hospitalar. Além disso, contribuiu para revisão e padronização das terminologias referentes às causas dos cancelamentos.

Conclui-se que a taxa de cancelamento cirúrgico do hospital em questão, em 2016, esteve elevada em relação à taxa de referência apresentada pelo programa CQH no qual a instituição em estudo está credenciada, porém, é importante destacar que, quando comparado a outras instituições de saúde, este estudo apresenta taxa inferior.

O mapeamento das causas do cancelamento cirúrgico instrumentaliza o enfermeiro gestor na adoção de estratégias, no processo decisório e ações integradas entre os diversos serviços relacionados ao fluxo do paciente cirúrgico, fazendo com que haja redução na taxa de cancelamento.

Como sugestão para a realização de novos estudos, acredita-se ser importante a análise do custo do cancelamento cirúrgico, da suspensão cirúrgica, com o respectivo itinerário até a realização do procedimento e ajustes em relação a modalidade de cancelamento cirúrgico por especialidade médica.

1. Moraes PGS, Pachêco NMD, Souza e Silva RG, Silva PCV. Clinical and organizational factors related to cancellation of surgical procedures. J Nurs UFPE on line 2017;11(7):2645-53.

https://periodicos.ufpe.br/revistas/revistaenfermagem/article/view/23436/19133 
2. Martins FZ, Dall'Agnol CM. Centro cirúrgico: desafios e estratégias do enfermeiro nas atividades gerenciais. Rev Gaúcha Enferm 2016;37(4):e56945. https://doi.org/10.1590/1983-1447.2016.04.56945

3. Stumm EMF, Maçalai RT, Kirchner RM. Dificuldades enfrentadas por enfermeiros em um centro cirúrgico. Texto Contexto Enferm 2006;15(3):464-71. https://doi.org/10.1590/S0104-07072006000300011

4. Dhafar KO, Ulmalki MA, Felemban MA, Mahfouz ME, Baljoon MJ, Gazzaz ZJ et al. Cancellation of operations in Saudi Arabian hospitals: Frequency, reasons and suggestions for improvements. Pak J Med Sci 2015;31(5):1027-32. https://doi.org/10.12669/pims.315.7932

5. Lobo LC, Brito CF, Sabino AS, Almeida MC. Taxa de suspensão de cirurgias no Brasil: uma revisão integrativa. REAC 2019;4:e497. https://doi.org/10.25248/reac.e497

6. Carvalho TA, Sobral CB, Marinho PML, Llapa-Rodriguez EOO, Campos MPA. Suspension of surgery at a university hospital. Rev SOBECC 2016;21(4):186-91. https://doi.org/10.5327/Z1414-4425201600040002

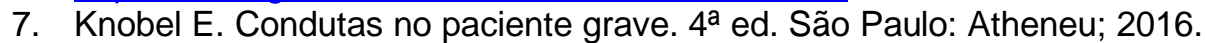

8. Gouveia Y. Revisão integrativa como estratégia para descrever os principais motivos, que levam a suspensão de cirurgias eletivas [Internet]. Uberlândia: Universidade Federal de Uberlândia; 2018 [cited 2020 junho 20]. 37 p. Available from: https://repositorio.ufu.br/bitstream/123456789/24236/1/Revis\%c3\%a3olntegrativaEstrat egica.pdf

9. Morgan W, Bernardino E, Wolff LDG. Implications of cancellation of surgery in a surgery department: a descriptive-exploratory study. Online Braz J Nurs 2019;9(1). http://www.objnursing.uff.br/index.php/nursing/article/view/j.1676-4285.2010.2591/585

10. Botazini NO, Toledo LD, Souza DMST. Cirurgias eletivas: cancelamento e causas. Rev Sobecc 2015;20(4):210-9.

11. Moreira LR, Xavier APR, Moreira FN, Souza LCM, Araujo OC, Santos TMB et al. Avaliação dos motivos de cancelamento de cirurgias eletivas. Enfermagem Revista 2016;19(2). http://periodicos.pucminas.br/index.php/enfermagemrevista/article/view/13156

12. Torri A. Estratégias para minimizar o cancelamento de cirurgias em um hospital escola [Internet]. Florianópolis: Universidade Federal de Santa Catarina; 2019 [citado 2020 jul 20]. 117 p. Disponível em: https://repositorio.ufsc.br/bitstream/handle/123456789/198129/PGCF0100D.pdf? sequence $=-1$ \&isAllowed $=y$

13. Santos GAAC, Bocchi SCM. Cancelamento de cirurgias eletivas em hospital público brasileiro: motivos e redução estimada. Rev Bras Enferm 2017;70(3):561-8. https://doi.org/10.1590/0034-7167-2016-0084

14. Prin M, Eaton J, Mtalimanja O, Charles A. High elective surgery cancellation rate in Malawi primarily due to infrastructural limitations. World J Surg 2018;42(6):1597-602. https://doi.org/10.1007/s00268-017-4356-1

15. Sampaio CEP, Gonçalves RA, Júnior HCS. Determinantes dos fatores da suspensão de cirurgia e suas contribuições para assistência de enfermagem. J Res Fundam Care Online 2016;8(3):4813-20. https://doi.org/10.9789/2175-5361.2016.v8i3.4813-4820

16. Abeldano RA, Coca SM. Tasas y causas de suspensión de cirugías en un hospital público. Enferm Universitaria -Mex 2016;13(2):107-13. https://doi.org/101016/j.reu.2016.03.005

17. Ladim FM, Paiva FDS, Fiuza MLT, Oliveira EP, Pereira JG, Siqueira IA. Análise dos fatores relacionados à suspensão de operações em um serviço de cirurgia geral de média complexidade. Rev Col Bras Cir 2009;36(4):283-7. https://doi.org/10.1590/S0100-69912009000400002

18. Santos G, Bocchi S. Cancellation of elective surgeries in a Brazilian public hospital: reasons and estimated reduction. Rev Bras Enferm 2017;70(3):535-5. https://doi.org/10.1590/0034-7167-2016-0084

19. Pinheiro S, Vasconcelos R, Oliveira J, Matos F, Tonini N, Alves D. Surgical cancellation rate: quality indicator at a public university hospital. REME Rev Min Enferm 2017;21:e1014. https://doi.org/10.5935/1415-2762.20170024

20. Bohrer CD, Marques LGS, Rigo DFH, Borges F, Vasconcelos RO et al. Causas de cancelamento cirúrgico em um hospital de ensino. Revista Eletrônica Gestão \& Saúde 2017;8(3). https://doi.org/10.18673/gs.v8i3.24123 